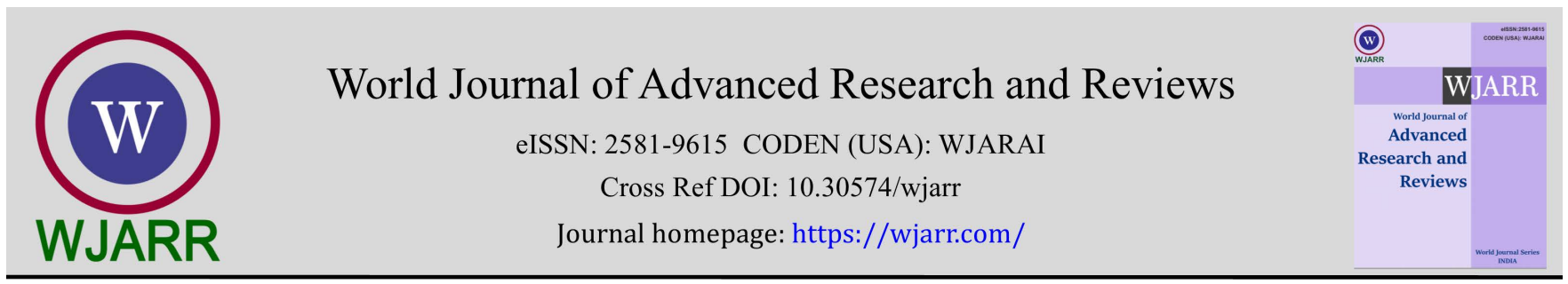

(RESEARCH ARTICLE)

\title{
The primary empty Sella: An endocrine study on 36 cases
}

\author{
Marwa Chiboub *, Fatma Mnif, Boubaker Ben Miloud, Dhouha Ben Salah, Nabila Rekik and Mohamed Abid \\ Endocrinology Unit, Department of Endocrinology, Hedi Chaker University Hospital, Sfax; Tunisia.
}

World Journal of Advanced Research and Reviews, 2021, 11(02), 124-129

Publication history: Received on 04 July 2021; revised on 09 August 2021; accepted on 11 August 2021

Article DOI: https://doi.org/10.30574/wjarr.2021.11.2.0371

\begin{abstract}
Introduction: The term primary empty sella (PES) makes reference to the herniation of the subarachnoid space within the sella turcica in patients with no history of pituitary tumor, surgery or radiotherapy.

Materials and Methods: We studied 36 patients; 27 females and 9 males. The mean age at diagnosis was 50, 4 years [21-80 years]. Most diagnoses were made by magnetic resonance imaging $(n=32)$. The anterior pituitary function was evaluated by basal hormonal measurements. Then, we had compared two groups of patients: G1(n=17), who had a pituitary disorder, and G2 ( $\mathrm{n}=10)$, patients without hormonal disease, in order to determine risk factors for endocrine dysregulation in PES.
\end{abstract}

Results: The reasons for ordering pituitary scans were: headaches (65\%), visual disturbances (32\%) and neurological symptoms in $8 \%$ of cases. Sixty-six point five per cent of women were multiparous. Diabetes, obesity and hypertension were found in $19.45 \%, 11 \%$ and $19.45 \%$ of the studied population respectively. Hyperprolactinemia was present in $17.24 \%$ of patients. Fifty-five percent of our patients had some degree of hypopituitarism. We didn't find any correlation in our study, between pituitary insufficiency and age, gender, weight, parity nor post-partum hemorrhage. Failure of lactation was significantly associated with a hormonal disorder. On the other hand, headache was negatively correlated with hormonal effects in PES.

Conclusion: PES was most commonly found in middle-aged multiparous women. In most patients, PES is a heterogeneous condition that ranges from hypopituitarism to various degrees of isolated GH deficiency, and which needs careful endocrine assessment, treatment and follow-up.

Keywords: Empty Sella; Primary; Sella turcica; Hypopituitarism

\section{Introduction}

The term, "empty sella," was first introduced by Busch in 1951 to describe "a peculiar anatomical condition, observed in 40 of 788 human cadavers, particularly females, characterized by a sella turcica with a diaphragma sellae incomplete or that forms only a small peripheral rim, with a pituitary gland not absent, but flattened in such a manner as to form a thin layer of tissue at the bottom of the sella turcica"[1]. Empty sella or arachnoidocele has been defined as the herniation of the subarachnoid space within the sella turcica, associated with elongated pituitary stalk and flattening of the pituitary gland [2]. Two types of empty sella should be distinguished. The first one and most common, called secondary empty sella (SES), usually results from a pituitary adenoma that shrinks after different treatments (surgery, radiotherapy or drugs) or spontaneous regression [3]. It may also result from postpartum pituitary necrosis (Sheehan syndrome) or lymphocytic hypophysitis. The second type of empty sella, called primary empty sella (PES), is unrelated to any known previous pathological process in the pituitary gland. PES may be a radiological finding in asymptomatic

\footnotetext{
${ }^{*}$ Corresponding author: Marwa Chiboub

Endocrinology Unit, Department of Endocrinology, Hedi Chaker University Hospital, Sfax, Tunisia.
} 
patients or it may be associated with variable clinical conditions such as neurological, visual and/or endocrine disorders [4]. Increased intracranial cerebrospinal fluid (CSF) pressure, in association with defects of the sellar diaphragm, are considered as the pathogenic factors of PES [2]. Both would be present in obesity and in multiparous women. Empty sella is defined as partial or total when less or more than $50 \%$ of the sella is filled with CSF respectively, with the gland thickness being $\backslash 2 \mathrm{~mm}$ in the latter case [5]. The widespread use of computed tomography (CT) and magnetic resonance imaging (MRI) has made PES a common incidental finding. According to data obtained from autopsies and neuroradiological exams, the presence of empty sella ranges from 5.5 to $35 \%$, with a female/male ratio of 4/1[5,6]. The aim of this multicenter retrospective study was to report clinical, radiological and hormonal data of patients with PES. Then, we will try to compare two groups of patients with and without pituitary insufficiency in order to determine risk factors for endocrine dysregulation in PES.

\section{Methods}

We retrospectively evaluated the clinical data of 36 patients (27 women, 9 men) from different sites in Tunisia. All patients had PES diagnosed by MRI $(n=32)$ or CT $(n=10)$. Four patients provided a plain radiograph of the sella turcica, ordered as part of routine endocrine testing. Clinical data was obtained from medical records according to individual diagnostic criteria. Basal endocrine evaluation was performed in most patients: free thyroxine 4 (FT4), thyroidstimulating hormone (TSH), cortisol, luteizing hormone ( $\mathrm{LH}$ ), follicle stimulating hormone (FSH), estradiol, testosterone (men), prolactin (PRL). Hormones were measured using commercially available kits used in routine testing at the labs of the university hospital of Sfax. Thyrotropin-releasing hormone (TRH) test was performed to rule out subclinical primary hypothyroidism, and Adrenocorticotropic hormone (ACTH) test was performed to rule out secondary adrenal insufficiency. Insulin hypoglycemia test was performed to evaluate the somatotropic axis. Secondly, we used a comparative study of 2 groups with PES, 17 patients with a hormonal impact (G1) and 10 patients who had no evidence of pituitary disorder (G2), in order to analyze the predictive factors of a pituitary disorder in PES. We analyzed the data using Statistical packages for social sciences 22 determining the means and proportions.

\section{Results}

Of 36 patients studied, 27 were females and 9 males (ratio F/M: 3/1). The average age at diagnosis was 50.4 years (2180 years). Associated diseases and clinical conditions were investigated: diabetes, hypertension, and obesity were found in $19.45 \%, 11 \%$, and $19.45 \%$ of patients, respectively. Multiparity was noted in 16 patients among the 26 married women (61.53\%). A grand multiparity was noted in 9 women (parity $>5$ ). Total empty sella was documented in 32 patients by pituitary imaging, while total empty sella was found in 30 patients (Fig 1). Enlarged sella was found in 4 patients on plain radiograph, which prompted a pituitary MRI in all of these patients. Radiological studies (CT or MRI) were ordered for various reasons.

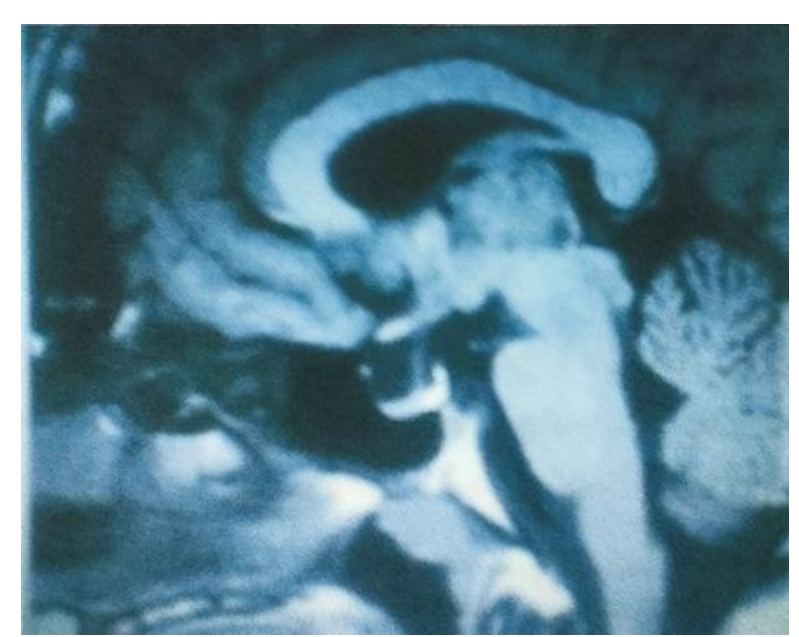

A

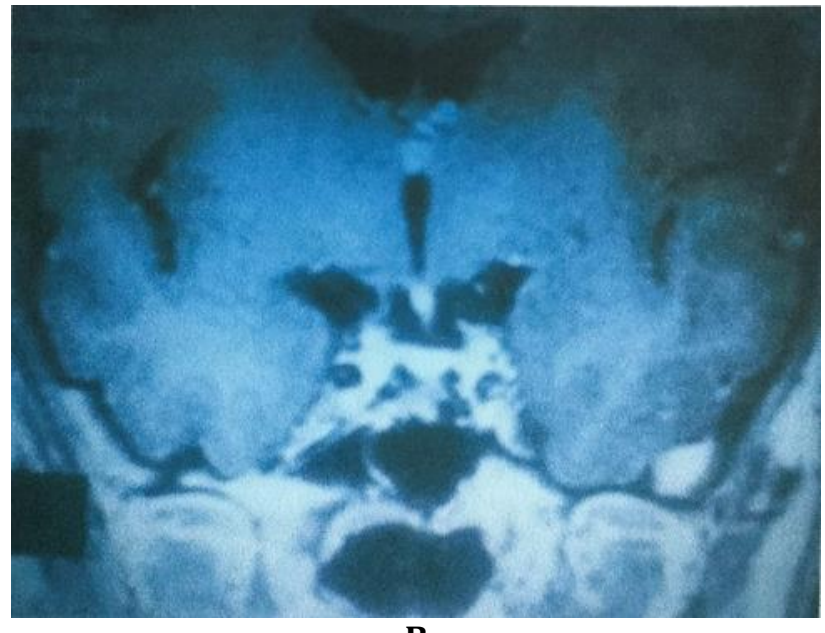

B

Figure 1 MRI of total empty sella: sagittal (a) and coronal (b) weighted T1 images

Headache has been reported as a symptom in $66.67 \%$ of patients. Neurological symptoms were noted in $8.3 \%$ of patients and visual disturbances were found in $30.56 \%$ of patients, including scotoma, hemianopsia and quadrantanopia ( 2 cases). Seven patients had papilledema on fundus examination and three had intracranial hypertension. No rhinorrhea was observed in our patients (Figure2). 


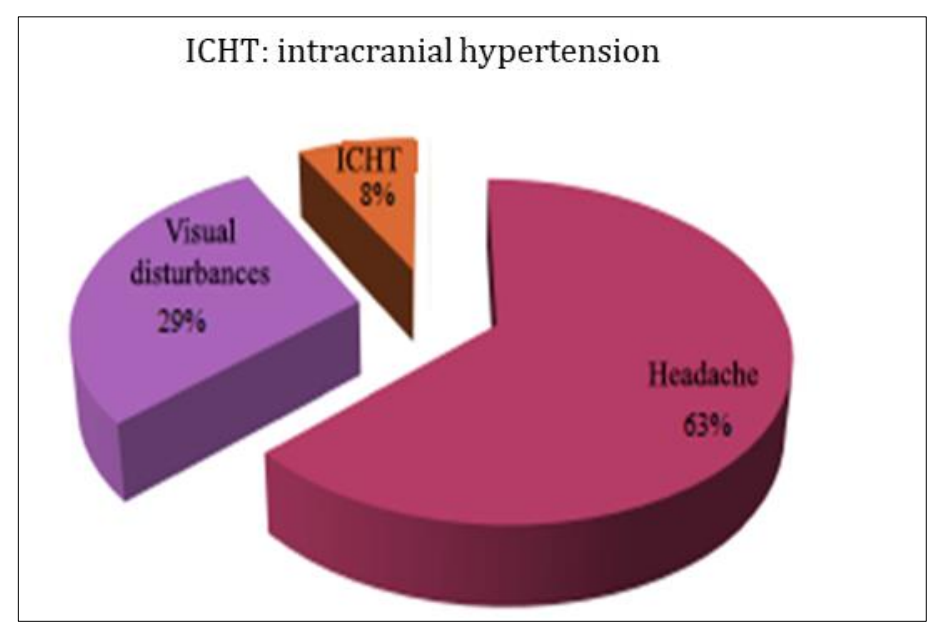

Figure 2 Discovery circumstance of primary empty sella

The most common symptoms secondary to hormonal irregularities in women were irregular menses in $80 \%$ ( $\mathrm{n}=22$ ) and galactorrhea in 26\% (n=7). Biochemical evaluation revealed hyperprolactinemia in $17.24 \%$ of patients. The mean PRL levels was $106.78 \mathrm{ng} / \mathrm{ml}$ (range 42-189) and only 2 cases had a prolactin level $>100 \mathrm{ng} / \mathrm{ml}$. The evaluation of the anterior pituitary function showed that $55.5 \%$ of patients $(n=20)$ had some degree of hypopituitarism, 4 of which were males. The anterior pituitary deficiency was dissociated in all cases. It involved the thyroid axis in $36 \%$ of cases(n=13). This deficit was isolated in three cases and associated with a secondary adrenal deficiency in 10 cases. A half of patients $53.6 \%$ of patients $(n=15)$ had secondary adrenal deficiency, $22.8 \%(n=8)$ had hypogonadotropic hypogonadism and 2 had diabetes insipidus. The insulin induced hypoglycemia test was indicated for only one patient to explore somatropin axis. The peak GH response was $>10 \mathrm{ng} / \mathrm{ml}$ which eliminated a GH deficiency. On the other hand, eight subjects had primary hypothyroidism without goiter. FT4 values were reduced with an average value of 3.87 pmol/l [0.03-8.8] associated to elevated TSH levels with an average value of $14.3 \mu \mathrm{U} / \mathrm{ml}$. Endocrine function tests were mentioned in table 1.

Table 1 Endocrine function tests in the 36 patients

\begin{tabular}{|l|c|}
\hline Endocrine function test & Average value \\
\hline FT4 $(\mathrm{pmol} / \mathrm{l})$ & $10.36[0.03-19.9]$ \\
\hline TSH $(\mu \mathrm{UI} / \mathrm{ml})$ & $4.66[0.14-50]$ \\
\hline TRH Test/TSH & 8.92 \\
\hline Cortisol $(\mu \mathrm{g} / \mathrm{L})$ & $91[1.31-171]$ \\
\hline ACTH $(\mathrm{pg} / \mathrm{ml})$ & $21.36[0.61-35.7]$ \\
\hline Female: FSH(UI/L) & $7.8[2.3-13]$ \\
LH(UI/L) & $5.4[1.5-14.8]$ \\
\hline Male: FSH(UI/L) & $5.7[0.6-9.8]$ \\
LH(UI/L) & $3.4[0.1-9.7]$ \\
\hline Estradiol $(\mathrm{pg} / \mathrm{ml})$ & $9.28[<9-10]$ \\
\hline
\end{tabular}

FT4: free thyroxine 4; TSH: thyroid-stimulating hormone; TRH test: thyrotropin-releasing hormone test; ACTH: Adreno corticotropic hormone; LH: luteizing hormone; FSH: follicle stimulating hormone.

Therapeutic abstention had concerned 10 patients, 7 of which have a normal hormonal balance. Hydrocortisone and levothyroxine replacement therapy were administered to 14 and 13 patients respectively with an average dose of 25 $\mathrm{mg} /$ day and $120 \mu \mathrm{g} /$ day respectively. Hormonal replacement treatment with sexual hormones was introduced to eight females (combined estrogen-progestogen). However, two men who had a hypogonadotropic hypogonadism were not treated. Hyperprolactinemia was treated with dopamine -agonist drugs in 5 cases (average dose, $3.75 \mathrm{mg}$ per day). The evolution of empty sella was marked by a persistence of headaches and visual disturbances in 17 and 8 patients, respectively. Besides, the analysis of the comparative study between the two groups: G1: PES with hormonal 
impairment and G2 without hormonal impact was able to demonstrate that age, sex, medical and gynecological history didn't intervene in pituitary function. However, a positive correlation between the failure of lactation and hormonal disease of PES was observed (Table 2).

Table 2 Comparison of epidemiological and medical characteristics of two groups

\begin{tabular}{|l|c|c|c|}
\hline & G1 (n=17) & G2(n=10) & $\mathbf{p}$ \\
\hline Age (year) & $50[21-80]$ & $49[22-66]$ & 0.74 \\
\hline Sex & $14 / 3$ & $5 / 5$ & NS \\
\hline Diabetes & 9 & 8 & 0.88 \\
\hline Obesity & 2 & 4 & 0.08 \\
\hline Parity & - & - & 0.3 \\
\hline Postpartum hemorrhage & 5 & 1 & 0.39 \\
\hline Failure of lactation & 6 & 5 & 0.049 \\
\hline \multicolumn{2}{|c|}{ P: p-value; NS: not significant }
\end{tabular}

Concerning the tumor syndrome, the presence of headaches was characteristic of group 2 ( $p=0.01)$ while there was no statistically significant correlation between the hormonal impairment and the presence of signs of intracranial hypertension or visual disturbances (table3).

Table 3 Consultation's reason into the 2 groups

\begin{tabular}{|l|c|c|c|}
\hline Consultation's reason & G1 & G2 & P value \\
\hline Headaches & 10 & 10 & 0.01 \\
\hline Visual disturbances & 5 & 5 & 0.57 \\
\hline Intracranial hypertension & 1 & 2 & NS \\
\hline Irregular menses & 13 & 3 & 0.08 \\
\hline Galactorrhea & 5 & 2 & NS \\
\hline
\end{tabular}

In addition, no correlation was evidenced between radiological findings and pituitary function.

\section{Discussion}

Primary empty sella is a common finding in autopsies and neuroradiological exams, ranging from 5.5 to 35 \%, and most commonly reported in females [7]. In our study, we found 27 women and 9 men with PES (3/1). Pregnancy may trigger the onset of PES. The pituitary volume doubles during pregnancy, especially in the case of multiple pregnancies. This may contribute to the herniation of the subarachnoid space in case of hypoplastic sellar diaphragm and/or CSF hypertension, even if it is moderate and temporary [5]. Our data show a high prevalence of PES in women with multiple pregnancies (61.53\%). PES has been associated with obesity in several reports [8,9]. It is believed that morbid obesity can induce hypercapnia, which would be associated with chronically elevated CSF pressure and induce herniation of the suprasellar subarachnoid space [7]. Some authors have documented a clear relationship between intra-abdominal, intrathoracic and intracranial pressure in obese patients [10]. In Our data, $11 \%$ of our patients were obese. Concerning other comorbidities, diabetes and hypertension were the two most frequently reported conditions affecting $19.45 \%$ of the study group.

Headache is one of the most prevalent symptoms in PES, reported in about $60-80 \%$ of cases [7] similar to our findings $(66.6 \%)$.

Saindaine.al evaluated incidental PES on MRI with idiopathic intracranial hypertension (IIH) and documented headache as non-specific to PES but related IIH to be a significant determinant of headaches [11]. Anyway, in patients with PES, 
headache may be hypothesized to be caused by the traction on vascular-meningeal structures in the sellar cavity, although there is no conclusive evidence. Visual disturbance was the second most common complaint presented in our study (30.5\%). It has been reported in 1.6-16\% of cases [7].Transient visual disturbance, nystagmus, diplopia and blurred vision were some of the commonly reported visual impairment. Around $2.6 \%$ were diagnosed to have optic nerve abnormalities like compression, atrophy or neuritis. Most of the visual defects are a result of the involvement of optic nerve, which means that PES is more than just an incidental finding [12]. As identified by Saindaine.al, visual defects cannot be characteristic of incidental PES, without eliminating the role of IIH. Although other studies have reported a lower prevalence of visual defects and suggested the importance of an ophthalmic assessment of PES patients with visual field defects [13]. Menstrual irregularities, galactorrhea and sexual disturbances were highly reported in our study, as an Italian research which reported a higher prevalence of menstrual and sexual dysfunction in PES[12]. The most common pituitary disorders associated with PES are hyperprolactinemia and GH deficiency. PRL levels are usually below $100 \mathrm{ng} / \mathrm{ml}$. Different degrees of hypopituitarism ranging from 8 to $60 \%$ have been reported. [7]. In fact, the literature on hypopituitarism in incidental PES is sparse. The assumed estimated prevalence is 52\% [38\%; 65\%][14]. Insufficiencies in multiple pituitary axes are more common, with an estimated prevalence of 30\% [19\%; 44\%] than isolated such events, with a prevalence of $21 \%$ [12\%; 44\%]. The somatotropic axis seems to be the most commonly affected, followed by the gonadotropic axis. In our study, $55.5 \%$ of patients had some degree of hypopituitarism. The most affected axis was the corticotropic axis followed by the thyroid axis. The somatotropic axis was not studied in most of our patients either because of the unavailability of a proper test, the absence of symptoms related to GH deficiency, or the absence of an intention to treat patients. Furthermore, eight subjects had primary hypothyroidism without goiter and two patients had primary adrenal deficiency. The association between primary thyroid diseases and PES has been reported by other authors $[15,16]$. This is an interesting point, as in some cases PES may be the result of an autoimmune process involving the pituitary gland. A recent study, however, suggests that autoimmunity does not play a major role in the pathogenesis of PES syndrome[17].

Across all studies, in order to analyse the predictive factors of a pituitary disorder in PES, a higher prevalence of hormonal disturbances was seen in women. In addition to female sex, a higher body mass index (BMI) and hypertension were considered as risk factors. Lupi et al. [15]investigated the association between overweight (BMI 25 to $<30 \mathrm{~kg} / \mathrm{m} 2$ ), obesity (BMI $\geq 30 \mathrm{~kg} / \mathrm{m} 2$ ) and pituitary insufficiency in PES. Hypopituitarism had been diagnosed in 24 of 39 (62\%) overweight patients and in 15 of 33 (45\%) obese patients, but in only 2 of $13(15 \%)$ patients with a normal weight. In contrast, we didn't find any correlation in our study, between pituitary insufficiency and age, gender, weight, parity nor postpartum hemorrhage. Failure of lactation was significantly associated with a hormonal disorder. On the other hand, headache was negatively correlated with hormonal effects in PES.

Treatment of patients with PES includes hormone replacement and dopamine agonists in patients with symptomatic hyperprolactinemia. Patients with signs and symptoms of severe intracranial hypertension, disabling headache, visual disturbances and rhinorrhea should be carefully evaluated for potential neurosurgical treatment. Asymptomatic patients may just remain under observation, as they are unlikely to develop hormonal and radiological changes during follow-up. As this is a retrospective cross-sectional study, no follow-up data could be obtained. However, because of the theoretical risk of progression, a regular endocrine, neuro-ophthalmological and radiological reassessment is recommended in the literature [7].

\section{Conclusion}

Patients with PES should always undergo an endocrine, neurological, and ophthalmological evaluation at presentation because of the very high incidence of these abnormalities. Endocrine screening should be followed by specific endocrine testing when hormonal abnormalities are suspected. All endocrine deficits should be treated with appropriate medical substitution. PES should not be considered as merely an incidental finding and clinicians should be aware of its varying presentations and aspects. Non-symptomatic cases do not require treatment, but periodical follow-up is strongly advised. Monitoring of therapies in patients with symptomatic ES does not differ from the other medical conditions in which those treatments are indicated.

\section{Compliance with ethical standards}

\section{Acknowledgments}

My sincere appreciation and thanks to my co-authors who supported me in the course of my research.

\section{Disclosure of conflict of interest}


The authors declare that there is no conflict of interest that could be perceived as prejudicing the impartiality of the research reported.

\section{Statement of informed consent}

Owing to the fact that study was retrospective and data was from records and not directly from the patients.

\section{References}

[1] Busch W. Die Morphologie der Sella turcica und ihre Beziehungen zur Hypophyse. Virchows Arch Pathol Anat Physiol Klin Med. 1951; 320(5): 437-458.

[2] Guitelman M, Garcia Basavilbaso N, Vitale M, Chervin A, Katz D, Miragaya K, Herrera J, Cornalo D, Servidio M, Boero L, Manavela M, Danilowicz K, Alfieri A, Stalldecker G, Glerean M, Fainstein Day P, Ballarino C, Mallea Gil MS, Rogozinski A. Primary empty sella (PES): a review of 175 cases. Pituitary. 2013; 16(2): 270-274.

[3] Guinto G, del Valle R, Nishimura E, Mercado M, Nettel B, Salazar F. Primary empty sella syndrome: the role of visual system herniation. Surg Neurol. 2002; 58(1): 42-7; discussion 47-48.

[4] Zada G, Lopes MBS, Mukundan S, Laws E. Empty Sella Syndrome. In: Zada G, Lopes M, Mukundan S, Laws E, editors. Atlas of Sellar and Parasellar Lesions. Cham, Switzerland: Springer. 2016; 489-492.

[5] De Marinis L, Bonadonna S, Bianchi A, Maira G, Giustina A. Primary empty sella. J Clin Endocrinol Metab. 2005; 90(9): 5471-5477.

[6] Sage MR, Blumbergs PC. Primary empty sella turcica: a radiological-anatomical correlation. Australas Radiol. 2000; 44(3): 341-348.

[7] Guitelman M, Garcia Basavilbaso N, Vitale M, Chervin A, Katz D, Miragaya K, Herrera J, Cornalo D, Servidio M, Boero L, Manavela M, Danilowicz K, Alfieri A, Stalldecker G, Glerean M, Fainstein Day P, Ballarino C, Mallea Gil MS, Rogozinski A. Primary empty sella (PES): a review of 175 cases. Pituitary. 2013; 16: 270-274.

[8] Yngrid N, David L, Roberto A, Maricela V. Silla turca vacía e hipopituitarismo. Revista Medica Herediana. 1998; 9(2): 84-88.

[9] Sugerman HJ, DeMaria EJ, Felton WL 3rd, Nakatsuka M, Sismanis A. Increased intra-abdominal pressure and cardiac filling pressures in obesity-associated pseudotumor cerebri. Neurology. 1997; (2): 507-511.

[10] Komatsu M, Kondo T, Yamauchi K, Yokokawa N, Ichikawa K, Ishihara M, Aizawa T, Yamada T, Imai Y, Tanaka K, et al. Antipituitary antibodies in patients with the primary empty sella syndrome. J Clin Endocrinol Metab. 1988; 67(4): 633-638.

[11] Saindane AM, Lim PP, Aiken A, Chen Z, Hudgins PA. Factors determining the clinical significance of an "empty" sella turcica. AJR Am J Roentgenol. 2013; 200(5): 1125-1131.

[12] Ekhzaimy AA, Mujammami M, Tharkar S, Alansary MA, Al Otaibi D. Clinical presentation, evaluation and case management of primary empty sella syndrome: a retrospective analysis of 10-year single-center patient data. BMC Endocr Disord. 2020; 20(1): 142.

[13] Maira G, Anile C, Mangiola A. Primary empty sella syndrome in a series of 142 patients. J Neurosurg. 2005; 103(5): 831-836.

[14] Auer MK, Stieg MR, Crispin A, Sievers C, Stalla GK, Kopczak A. Primary Empty Sella Syndrome and the Prevalence of Hormonal Dysregulation. Dtsch Arztebl Int. 2018; 115(7): 99-105.

[15] Luboshitzky R, Barzilai D. Primary empty sella syndrome and hypopituitarism associated with primary hypothyroidism. J Endocrinol Invest. 1981; 4(2): 213-216.

[16] LaFranchi SH, Hanna CE, Krainz PL. Primary hypothyroidism, empty sella, and hypopituitarism. J Pediatr. 1986; 108(4): 571-573.

[17] Del Monte P, Foppiani L, Cafferata C, Marugo A, Bernasconi D. Primary "empty sella" in adults: endocrine findings. Endocr J. 2006; 53(6): 803-809. 